\title{
Research Paper: Effectiveness of Kinesiotaping and Conventional Physiotherapy on Patients With Non- Specific Chronic Low Back Pain
}

\author{
Hooman Mohammadalizade ${ }^{1}$, Zahra Mosallanezhad ${ }^{1 *}$, Noureddin Karimi ${ }^{1}$, Hamide Miri Abyane ${ }^{1}$, Enayatollah Bakhshi ${ }^{1}$ (D)
}

1. Department of Physiotherapy, University of Social Welfare and Rehabilitation Sciences, Tehran, Iran.

\begin{tabular}{|l|l|l|l}
\hline $\begin{array}{c}\text { use vour devict to san } \\
\text { and read the article online }\end{array}$ & $\begin{array}{l}\text { Citation Mohammadalizade H, Mosallanezhad Z, Karimi N, Miri Abyane H, Bakhshi E. Effectiveness of Kinesiotaping and } \\
\text { Conventional Physiotherapy on Patients With Non-Specific Chronic Low Back Pain. Physical Treatments. 2018; 7(4):233-240. } \\
\text { http://dx.doi.org/10.32598/ptj.7.4.233 }\end{array}$ \\
doi: : http://dx.doi.org/10.32598/ptj.7.4.233
\end{tabular}

Funding: See Page 238

\section{(c) (i) (8)}

Article info

Received: 10 Aug 2017

Accepted: 02 Dec 2017

Available Online: 01 Jan 2018

Keywords:

Kinesiotape,

Physiotherapy, Non-

specific chronic low back pain, Disability

\begin{abstract}
A B S T RA C T
Purpose: Low Back Pain (LBP) is among the most common reasons of medical visits worldwide. It can cause limited movements, long lasting disability, and low quality of life and work. Thus, studying the efficacy of conservative treatment strategies to help patients with LBP is very important. The present study aimed to evaluate the effect of kinesiotaping in addition to conventional physiotherapy on pain and disability in patients with non-specific chronic LBP
\end{abstract}

Methods: Of the non-specific chronic LBP patients who referred to a private physiotherapy clinic in Karaj City, Iran, patients with the age range of 20-55 years were included in this study, using simple random sampling method. Before the onset of intervention, patients on the waiting list for treatment for 2 weeks were considered as the controls. The intervention program performed in 10 sessions ( 2 weeks), comprising physiotherapy (ultrasound, hot pack, electrical stimulation and stability exercise) in addition to kinesiotaping which was applied on every session. Pain severity (using Visual Analog Scale) and disability (using Oswestry) were evaluated before, during and after the intervention. The repeated measures ANOVA was used for statistical analysis.

Results: Overall, 15 patients with the Mean \pm SD age of $39 \pm 8.9$ years and the body mass index of $26.92 \pm 5.46 \mathrm{~kg} / \mathrm{m}^{2}$, participated in the study. During the time that we considered as the period of control, assessments were performed and the results regarding pain and disability showed no significant changes $(\mathrm{P}>0.05)$. Changes in the mean score of pain severity were significant ( $P>0.001$ ); it altered from 7.33 before the intervention to 1.80 after the intervention. Disability based on the Oswestry scale changes were also significant $(\mathrm{P}>0.03)$; it changed from $50 \%$ before the intervention to $11 \%$ after that.

Conclusion: Combined kinesiotaping and conventional physiotherapy can decline pain and disability in patients with LBP.

\section{* Corresponding Author:}

Zahra Mosallanezhad, PhD

Address: Department of Physiotherapy, University of Social Welfare and Rehabilitation Sciences, Tehran, Iran.

Phone: +98 (21) 22180039

E-mail: zmosallanezhad@yahoo.com 


\section{Highlights}

- Applying of Kinesio-taping and conventional physiotherapy in patients with non-specific chronic LBP, can cause reduction in reduction of pain in patient with non-specific chronic low back pain.

- Physiotherapy interventions along with Kinesio-taping can significantly improve LBP and disability in patients with non-specific chronic low back pain.

- Kinesio-taping is a safe and effective intervention in management of patients with non-specific chronic low back pain. It is supposed to stabilize muscles and give awareness of muscle engagement, known as proprioception.

\section{Plain Language Summary}

Most of adults in the general population have experienced Low Back Pain (LBP) at least once in their lifetime. It is among the major causes of disability. Numerous interventions for treating patients with chronic LBP are available, but most of them have a modest effect on reducing pain and disability. Different types of physiotherapy techniques are among non-invasive interventions, used to increase the therapeutic effects and patient satisfaction. Kinesio-taping is one of these methods recommended for the clinical management of patients with musculoskeletal pain, particularly cases of LBP. Kinesio tape is supposed to stabilize muscles and give awareness of muscle engagement, known as proprioception. In addition, some claim that it slightly lifts the skin away from the muscle, which is supposed to help reduce swelling and promote blood flow to the muscle. The results of present study indicate that physiotherapy interventions along with Kinesio-taping can significantly improve LBP and disability in patients with non-specific chronic LBP.

The mechanism of its action is providing protection and support for damaged muscles and joints and reduces pain by pulling the skin, and improving blood and lymph circulation. This study finding is important because it could provide a scientific evidence for efficacy of physiotherapy techniques along with Kinesio-taping in managing LBP.

\section{Introduction}

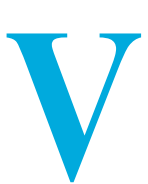

arious Low Back Pain (LBP) are highly frequent in society and is spreading worldwide [1-4]. LBP is among the major causes of disability and $70 \%$ to $80 \%$ of adults in the general population have experienced LBP at least once in lifetime $[2,4]$. Studies indicate that about $10 \%$ to $15 \%$ of acute LBP will turn into chronic LBP. The frequency of chronic LBP is about $23 \%$ and in general, $11 \%$ to $12 \%$ of the population suffer from disabilities caused by LBP [1-3]. The consequences of high prevalence of LBP are huge direct and indirect costs [2, 3]. Introduction and application of caring strategies, especially effective methods in relieving pain and disability in chronic LBP are of great importance [2].

Numerous interventions for treating patients with chronic LBP are available, but most of them have a modest effect on reducing pain and disability [1]. Complications caused by extensive and long-term use of home remedies call for non-medicinal treatment approaches. However, aggressive and combined treatments like op- eration are not recommended for these patients [3]. Different types of physical movements and physiotherapy techniques are among non-invasive interventions, used to increase the therapeutic effects and patient satisfaction. Kinesio-taping is one of these methods recommended for the clinical management of patients with musculoskeletal pain, particularly cases of LBP [1-8].

Kinesio-taping is a modern treatment which is popular, especially among athletes and used to repair damaged tissues due to exercise [3]. Kinesio-taping technique has been effective for the treatment of various musculoskeletal disorders, including injuries, and in particular, damages caused by exercise. The review and original studies conclude that kinesio-taping not only has a widespread use in musculoskeletal disorders, but also has benefits for patients with acute pain control and improves the timely muscle performance. However, few studies are available on the application of this method and even less evidence to support the use of Kinesio-taping in chronic musculoskeletal disorders [5-7, 9-13].

Although the use of Kinesio-taping in treatment of chronic musculoskeletal disorders is recommended in 
some studies, its exact mechanism of action on improving these disorders is not fully understood. Cass et al. witnessed a blood flow increase in adherent areas and this hypothesis has been supported by Doppler ultrasound [14]. Kask claimed that Kinesio-taping protects damaged muscles and joints and reduces pain by pulling the skin, thus, improves blood and lymph circulation $[2,7,13]$.

In a review study by Kalron, the efficacy of Kinesiotaping was not proved in motor disorders and a wide range of pathological disabilities [15]. A meta-analysis by Kumbrink concludes that in spite of the useful role of Kinesio-taping in improving power, range of motion, and proprioception in athletes, there was little evidence to support this approach in preventing sports injuries, compared to other methods, in general [7]. Because of high prevalence of LBP globally and also Iranian population [16], and lack of consensus among studies over the effect of non-invasive interventions in chronic LBP, this study aimed to evaluate the effect of using Kinesio-taping along with conventional physiotherapy on reducing pain and disability in patients with non-specific chronic LBP.

\section{Materials and Methods}

This was a quasi-experimental and single-group study which included patients referring to a private physiotherapy clinic in Karaj City, Iran in 2013-14. Considering the inclusion criteria (aged 20 to 55 years, having non-specific chronic LBP for at least 3 months, and being capable of receiving physiotherapy), 15 patients were selected with simple random sampling method and entered in the study after signing a written informed consent. Sample size required for research with $95 \%$ confidence and $80 \%$ power was calculated based on the sample size calculation formula for analytical studies and according to data of a preliminary study.

During ten sessions (in two weeks), the conventional physiotherapy, including stability exercises, ultrasonic waves, hot pack and electrical stimulation were applied to patients. In addition, the Kinesio-taping intervention was applied for them in each session. Kinesio tapes were attached to the outer abdominal muscles, in a specific way on each muscle, to support abdominal internal movements, and straighten muscles of the lumbar spine. To apply Kinesio-taping method to the external abdominal muscle, the distance from lower iliac crest and inguinal ligament to ribs 5 th to 12 th was measured while the arm, knee and the hip on the same side were flexed, and the hip was abducted.

Then the muscle was put in the resting position and the kinesio tape was attached to the inguinal ligament and iliac crest area without any tension, then the kinesio tape was attached along the length he muscle with $10 \%$ stretch. For intra-abdominal muscles, the distance from lower iliac crest towards ribs 8th to 12th was measured with the knees bending and turned on the same side and the upper trunk turned on the opposite side. Then, one end of the kinesio tape was attached to the beginning of the muscle in rest and without any tension.

Then the muscle was repositioned along the length and tapes were attached with 10\% stretch. For Kinesio-taping of posterior extensor muscle, first the distance from the sacrum to 12 th rib was measured with the trunk in flexion position and then, one end of the kinesio tape was attached to the upper sacrum margin without any tension with the trunk in the resting position (standing mode). Once again, the muscle was placed longitudinally (with patient bending), and the kinesio tape, which was in the form of $\mathrm{Y}$, was attached along to the muscle length to the 12 th thoracic vertebrae with $10 \%$ stretch [17-23]. In addition to recording the patient's demographic information at the beginning of the project, the desired outcomes of each session before the treatment were evaluated. The intensity of pain and degree of disability were assessed by Visual Analogue Scale (VAS) and Oswestry Disability Index (ODI), respectively.

Table 1. The mean score of underlying variables of the subjects

\begin{tabular}{cc}
\hline Variable & Mean \pm SD \\
\hline Age, $y$ & $39.00 \pm 8.90$ \\
Weight, $\mathrm{kg}$ & $76.07 \pm 15.43$ \\
Height, $\mathrm{cm}$ & $168.8 \pm 14.24$ \\
BMI, $\mathrm{kg} / \mathrm{m}^{2}$ & $26.92 \pm 5.46$ \\
Duration of the disease, mon & $20.07 \pm 11.12$ \\
\hline
\end{tabular}




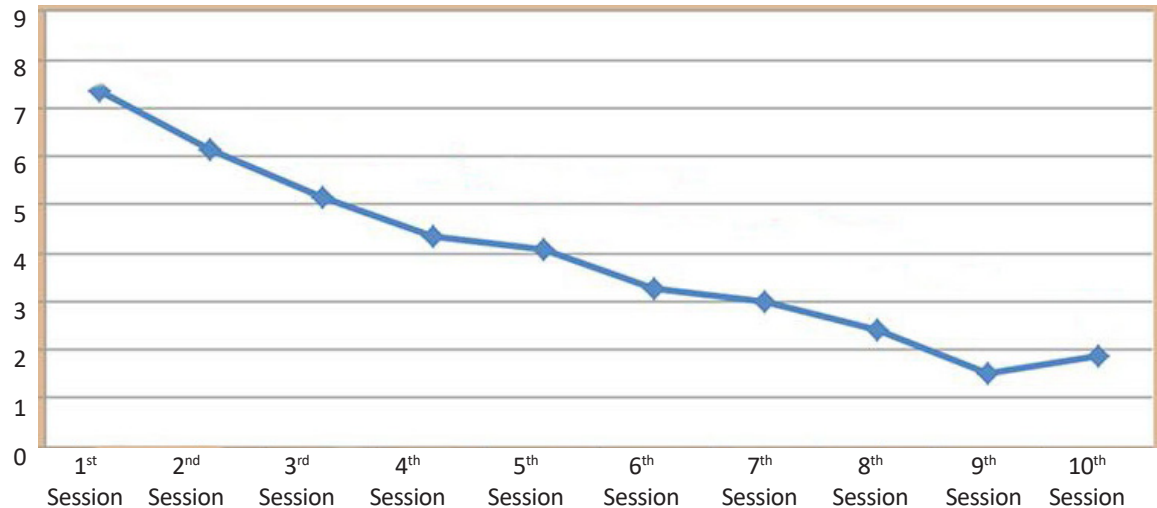

Figure 1. Mean score of pain in patients in 10 times measurement

PHYSICAL TREA $\|$ MENTS

Eventually, the obtained data were analyzed statistically. Normality of the study variables was calculated using normal distribution test and then the desired variables were compared using repeated measures ANOVA and the degree of the changes was also examined using the graph.

\section{Results}

Table 1 presents the mean underlying variables such as age, weight, height, Body Mass Index (BMI), and the duration of the disease. The distribution of underlying variables was proportional to the normal distribution $(\mathrm{P}>0.05)$. Figure 1 shows the mean score of pain in 10 times measurement. The mean score of pain reduced from 7.33 at the start of treatment to 1.80 at the $10^{\text {th }}$ session which was a significant reduction in pain intensity $(\mathrm{P}<0.001)$.

Figure 2 depicts the mean scores of ODI in 10 times measurement. Mean score of disability from 0.50 at the beginning of treatment reached 0.11 at the 10th session (in the sense of improving ability). Based on the aesthetic criteria in these patients, the reduction in disability was significant $(\mathrm{P}<0.03)$.

\section{Discussion}

Overall, results indicate that physiotherapy interventions along with Kinesio-taping can significantly improve LBP and disability in patients with non-specific chronic LBP. Studies report the beneficial effects of Kinesio-taping in treatment of various musculoskeletal disorders [5, 7, 9-12].

The current study indicates reduction of pain with the use of Kinesio-taping and conventional physiotherapy in patients with non-specific chronic LBP. This finding is consistent with previous studies which showed reduced skeletal pain, improvement of muscle activity, and acute pain control in physical rehabilitation of patients with chronic LBP by Kinesio-taping [5, 6]. Kalron in a review study reported immediate reduction of pain in musculoskeletal disorders using this method [13].

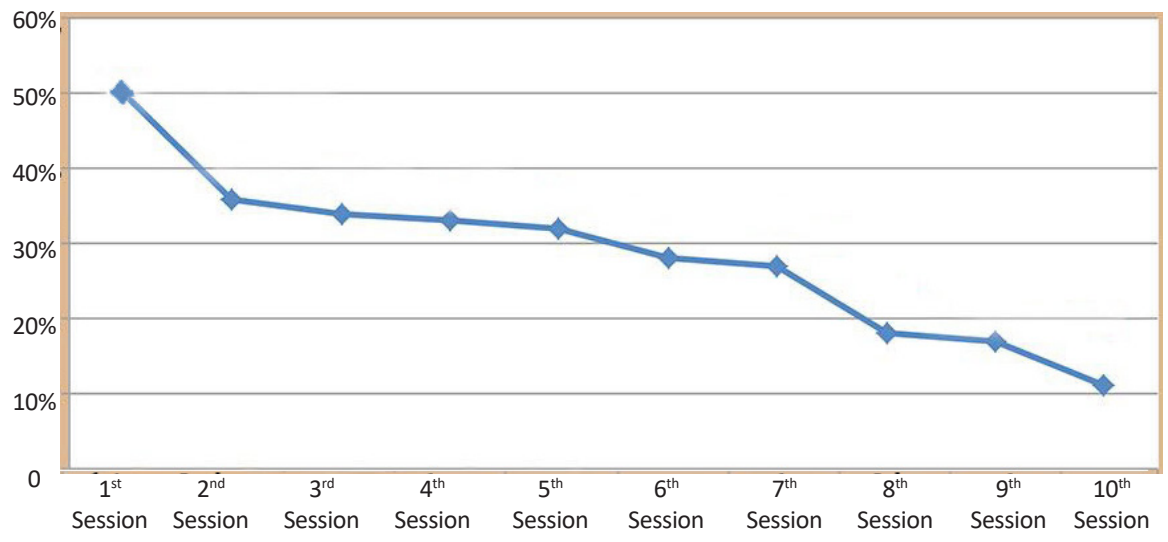

Figure 2. Mean scores of ODI in 10 times of measurements in the study subjects 
In a review study by Mostafavifar, he conclude that Kinesio-taping can significantly improve pain and disability in patients with acute injury on the cervical vertebrae and these effects remain within 24 hours, in addition to damage time. There is also a significant improvement in short-term pain in the lumbar area in patients with chronic LBP under Kinesio-taping treatment, with and without exercise [9].

A similar study conducted by Added reported beneficial effects of adding kinesiology intervention to physiotherapy in all clinical outcomes including the intensity of the pain at the beginning and in 5 weeks, 3 months and 6 months after conducting the intervention in patients with non-specific chronic LBP [1]. Studies indicate that Kinesio-taping is not only effective in reducing the severity of LBP, but also effective in reducing pain in other parts of the body, including mechanical cervical pain [11, 17], lumbar spine [2, 16, 18, 19], cervical collars [10], knee patella pain syndrome [20], injured Achilles tendon [21] shoulder impingement syndrome [12], and meralgia paresthetica [22]. Results of some studies like Maurice review study [8], metaanalysis of Williams [7] and Admczyk study [2] were inconsistent with the current study.

ODI results in the current study proved improvement of disability due to physiotherapy intervention along with Kinesio-taping. Some studies did not report the effectiveness of Kinesio-taping in improving disabilities in patients with LBP. Kalron review study [15], Mostafavifar study [9], and Admczyk study [2] are among those studies.

In various studies on different populations, it was shown that care programs like physical activity and physiotherapy techniques as well as manual methods like Kinesio-taping in patients with LBP have improved the pain and disability of the patients $[2,4$, 23]. Overall, the literature review reveals that a healthy lifestyle and use of conservative treatment are effective and beneficial for treating LBP and invasive treatments like operation are not recommended [4].

Kinesio-taping is a modern treatment for various musculoskeletal disorders, including LBP. Numerous studies support the effectiveness of this therapeutic approach on patients with musculoskeletal disorders, especially LBP, worldwide. However, the number of definitive and appropriate high-quality investigations on the use of Kinesio-taping in musculoskeletal disorders are very limited [3, 23-26]. Although the Kinesio-taping method is used by physicians for a wide range of disorders, and particularly in patients with chronic LBP, many studies that re- ported Kinesio-taping benefits, were potentially biased, case reports, or without controls [2].

Further investigations on different populations are required to explore the effectiveness of Kinesio-taping on improving the symptoms of musculoskeletal disorders. There is no consensus among prior studies on the benefits and effectiveness of this kind of therapy. This was a quasi-experimental study under controlled conditions, with random selection of subjects with the control period. The therapist and the evaluator were different in this research and it was a one-way blinded study. The therapist performed all treatment sessions, and evaluation of patients in each treatment cycle was carried out by the research partner separately from the other sessions. Evaluator mastered the methods of evaluation and he had enough skill in that case.

Considering the importance of using valid measuring instruments, the validity of questionnaires in this study were examined. The results of these studies showed that Visual Analogue Scale for Pain (VAS) and Roland Morris Questionnaire are valid instruments which are commonly used to assess the outcomes of the disease in patients with LBP. The results also indicated that these questionnaires had appropriate reliability and validity to assess pain and disability in patients with LBP [27-29].

Considering the limitations in the inclusion of specific individuals in this study and the existence of specific conditions related to the type of study, our study findings cannot be generalized to other patients in different situations. In this study, treatment and evaluation were applied by two separate individuals to reduce biasedness. Future studies are suggested to separate control group and considering the effect of placebo treatment. It is recommended to conduct similar interventions (with similar tapes but without placebo therapeutic effects) for the control group. It is also suggested that in future studies, synergistic effects of Kinesio-taping along with other non-invasive and less complicated treatment methods like special sports exercises be examined and compared in patients with chronic LBP and other musculoskeletal disorders.

Overall, the current study indicate that the application of Kinesio-taping technique along with conventional physiotherapy interventions in patients with non-specific chronic LBP decreases severity of LBP and reduces its disability.

The results of reviewed studies indicate no side effects for Kinesio-taping. Moreover, the results of this study is in line with other studies about the positive effects of Kinesio-taping. This kind of therapy can reduce pain, 
increase proprioception, increase blood and lymph circulation, and helps improve muscle and tendon function, without limiting the range of motion. Thus, Kinesio-taping along with conventional physiotherapy treatments is recommended for patients with LBP.

\section{Ethical Considerations}

\section{Compliance with ethical guidelines}

The study design and methodology were approved by the Ethics Committee of the University of Social Welfare and Rehabilitation Sciences.

\section{Funding}

This research did not receive any specific grant from funding agencies in the public, commercial, or not-forprofit sectors.

\section{Conflict of interest}

The authors certify that they have no affiliation with or involvement in any organization or entity with any financial interest, or non-financial interest in the subject matter or materials discussed in this manuscript.

\section{Acknowledgments}

The authors would like to express their special thanks to the patients who participated in the study and the physiotherapist involved in the assessment. In addition, we are grateful of the University of Social Welfare and Rehabilitation Sciences (Physiotherapy Department) and Hooman Physiotherapy Clinic for their executive support.

\section{References}

[1] Castro-Sánchez AM, Lara-Palomo IC, Matarán-Pe-arrocha GA, Fernández-Sánchez M, Sánchez-Labraca N, Arroyo-Morales $\mathrm{M}$. Kinesio-taping reduces disability and pain slightly in chronic non-specific low back pain: A randomised trial. Journal of Physiotherapy. 2012; 58(2):89-95. [DOI:10.1016/ S1836-9553(12)70088-7]

[2] Balagué F, Mannion AF, Pellisé F, Cedraschi C. Non-specific low back pain. Lancet. 2012; 379(9814):482-91. [DOI:10.1016/ S0140-6736(11)60610-7]

[3] Geiasi F, Akbari A, Sangtarash F. [The effect of stabilization method and Williams exercise on improvement of functions in patients with mechanical chronic low back pain (Persian)]. Journal of Shahrekord University of Medical Sciences. 2007; 8(4):21-8.
[4] Paoloni M, Bernetti A, Fratocchi G, Mangone M, Parrinello L, De Pilar Cooper M, et al. Kinesio-taping applied to lumbar muscles influences clinical and electromyographic characteristics in chronic low back pain patients. European Journal of Physical and Rehabilitation Medicine. 2011; 47(2):237-44. [PMID]

[5] Kask A, Wallis J. The latest kineiso taping method. Ski Journal. 2002; 47(2):237-44.

[6] kumbrink B. K Taping: An Illustrated Guide - Basics - Techniques - Indications. New York: Springer; 2011

[7] Morris D, Jones D, Ryan H, Ryan CG. The clinical effects of Kinesio Tex taping: A systematic review. Physiotherapy: Theory and Practice. 2013; 29(4):259-70. [DOI:10.3109/0959 3985.2012.731675] [PMID]

[8] Mostafavifar M, Wertz J, Borchers J. A systematic review of the effectiveness of Kinesio-taping for musculoskeletal injury. The Physician and Sportsmedicine. 2012; 40(4):33-40. [DOI:10.3810/psm.2012.11.1986] [PMID]

[9] Karatas N, Bicici S, Baltaci G, Caner H. The effect of Kinesiotape application on functional performance in surgeons who have musculo-skeletal pain after performingsurgery. Turkish Neurosurgery. 2012; 22(1):83-9. [DOI:10.5137/1019-5149. JTN.5377-11.1] [PMID]

[10] González-Iglesias J, Fernández-de-Las-Pe-as C, Cleland JA, Huijbregts P, Del Rosario Gutiérrez-Vega M. Shortterm effects of cervical Kinesio-taping on pain and cervical range of motion in patients with acute whiplash injury: A randomized clinical trial. Journal of Orthopaedic \& Sports Physical Therapy. 2009; 39(7):515-21. [DOI:10.2519/ jospt.2009.3072]

[11] Kaya E, Zinnuroglu M, Tugcu I. Kinesio-taping compared to physical therapy modalities for the treatment of shoulder impingement syndrome. Clinical Rheumatology. 2011; 30(2):201-7. [DOI:10.1007/s10067-010-1475-6] [PMID]

[12] Yap E. Myofascial pain-an overview. Annals-Academy of Medicine Singapore. 2007; 36(1):43-8. [PMID]

[13] Bronfort G, Haas M, Evans RL, Bouter LM. Efficacy of spinal manipulation and mobilization for low back pain and neck pain: A systematic review and best evidence synthesis. The Spine Journal. 2004; 4(3):335-56. [DOI:10.1016/j. spinee.2003.06.002] [PMID]

[14] Kalron A, Bar-Sela S. A systematic review of the effectiveness of Kinesio-taping - Fact or fashion? European Journal of Physical and Rehabilitation Medicine. 2013; 49(5):699-709. [PMID]

[15] Mohseni-Bandpei MA, Ahmad-Shirvani M, Golbabaei N, Behtash H, Shahinfar Z, Fernández-de-las-Pe-as C. Prevalence and risk factors associated with low back pain in Iranian surgeons. Journal of Manipulative and Physiological Therapeutics. 2011; 34(6):362-370. [DOI:10.1016/j. jmpt.2011.05.010] [PMID]

[16] Saavedra-Hernández M, Castro-Sánchez AM, ArroyoMorales M, Cleland JA, LaraPalomo IC, Fernández-de-LasPe-as C. Short-term effects of Kinesio-taping versus cervical thrust manipulation in patients with mechanical neck pain: A randomized clinical trial. Journal of Orthopaedic \& Sports Physical Therapy. 2012; 42(8):724-730. [DOI:10.2519/ jospt.2012.4086]

[17] Hwang-Bo G, Lee JH. Effects of Kinesio-taping in a physical therapist with acute low back pain due to patient han- 
dling: A case report. International Journal of Occupational Medicine and Environmental Health. 2011; 24(3):320-3. [DOI:10.2478/s13382-011-0029-8]

[18] Lee JH, Yoo WG. Application of posterior pelvic tilt taping for the treatment of chronic low back pain with sacroiliac joint dysfunction and increased sacral horizontal angle. Physical Therapy in Sport. 2012;13(4):279-85. [DOI:10.1016/j. ptsp.2011.10.003] [PMID]

[19] Akbaş E, Atay AO, Yüksel I. The effects of additional Kinesiotaping over exercise in the treatment of patellofemoral pain syndrome. Acta Orthopaedica et Traumatologica Turcica. 2011; 45(5):335-41. [DOI:10.3944/AOTT.2011.2403.] [PMID]

[20] Lee JH, Yoo WG. Treatment of chronic Achilles tendon pain by Kinesio-taping in an amateur badminton player. Physical Therapy in Sport. 2012; 13(2):115-9. [DOI:10.1016/j. ptsp.2011.07.002] [PMID]

[21] Chang HY, Wang CH, Chou KY, Cheng SC. Could forearm Kinesio-taping improve strength, force sense, and pain in baseball pitchers with medial epicondylitis? Clinical Journal of Sport Medicine. 2012; 22(4):327-33. [DOI: 10.1097/ jsm.0b013e318254d7cd]

[22] George SZ, Zeppieri G, Cere AL, Cere MR, Borut MS, Hodges MJ, et al. A randomized trial of behavioral physical therapy interventions for acute and sub-acute low back pain (NCT00373867). Pain. 2008, 140(1):145-57. [DOI:10.1016/j. pain.2008.07.029] [PMID] [PMCID]

[23] Bogduk N. Management of Chronic Low Back Pain. Medical Journal of Australia. 2004; 14(2):79-83. [PMID]

[24] Mostafavifar M, Wertz J, Borchers J. A systematic review of the effectiveness of Kinesio-taping for musculoskeletal injury. The Physician and Sportsmedicine. 2012; 40(4):33-40. [DOI:10.3810/psm.2012.11.1986]

[25] Halseth T, McChesney JW, DeBeliso M, Vaughn R. The effect of Kinesio-taping on proprioception at the ankle. Journal of Sports Science and Medicine. 2004; 3:1-17. [PMID] [PMCID]

[26] Vincent JI, Macdermid JC, Grewal R, Sekar VP, Balachandran D. Translation of oswestry disability index into Tamil with cross cultural adaptation and evaluation of reliability and validity. The Open Orthopaedics Journal. 2014; 8:11-9. [DOI:10.2174/1874325001408010011] [PMID] [PMCID]

[27] Payares K, Lugo LH, Morales V, Londono A.Validation in Colombia of the Oswestry disability questionnaire in patients with low back pain. Spine. 2011; 36(26):E1730-1735. [DOI:10.1097/BRS.0b013e318219d184] [PMID]

[28] Newman AN, Stratford PW, Letts L, Spadoni G. A Systematic Review of Head-to-Head Comparison Studies of the Roland-Morris and Oswestry Measures' Abilities to Assess Change. Physiotherapy Canada. 2013; 65(2):160-6. [DOI:10.3138/ptc.2012-12] 
